To appear in :

Physica Status Solidi Rapid Research Letters

Manuscript : 10.1002/pssr.202000534

\title{
How to Identify Lone Pairs, van der Waals Gaps and Metavalent Bonding using Charge and Pair Density Methods: From Elemental Chalcogens to Lead Chalcogenides and Phase Change Materials.
}

\author{
Jean-Yves Raty*1 ${ }^{*}$ Carlo Gatti ${ }^{2}$, Carl-Friedrich Schön ${ }^{3}$ and Matthias Wuttig ${ }^{3,4,5}$
}

1. CESAM and Condensed Matter Physics, B5, Université de Liège, B4000 Sart-Tilman, Belgium

2. CNR-SCITEC Istituto di Scienze e Tecnologie Chimiche 'Giulio Natta', via Golgi Section, via Golgi 19, Milano 20133, Italy.

3. Institute of Physics (IA), Physics of Novel Materials, RWTH Aachen University, 52056 Aachen, Germany

4. Jülich-Aachen Research Alliance (JARA FIT and JARA HPC), RWTH Aachen University, 52056 Aachen, Germany

5. PGI 10 (Green IT), Forschungszentrum Jülich GmbH, 52428 Jülich, Germany

E-mail: jyraty@uliege.be

Keywords : Phase Change Materials, Chalcogenides, Electronic Structure, Lone Pair, Metavalent Bonding, Ab Initio, MVB, van der Waals, Lead Oxide, Lead Telluride, Ge2Sb2Te5.

Lone pairs explain the structure of many molecular solids, as well as the chain-like or layered structures encountered in many chalcogenide crystals. Such chalcogenides have enabled a plethora of applications, including phase change memories, thermoelectrics, topological insulators or photoconductors. In many of these solids, lone pairs also have been invoked to explain the unconventional material properties. The presence of so-called van der Waals gaps in several layered chalcogenides, as well as their low thermal conductivity have also been linked to lone pairs. However, for some of these systems, a second, presumably competing view of bonding has been proposed, where atoms are held together across the interlayer spacing by shared electrons. To clarify this situation, we reinvestigate several systems theoretically, in which the role of lone pairs has been frequently emphasized. By comparing the charge and electron localization analysis in terms of a Hartree-Fock like pair density obtained from Kohn Sham DFT, we verify that the structure of several chalcogenides is governed by the presence of lone pairs, while others are not. As an example, crystalline $\mathrm{Se}$ is demonstrated to form a structure, where two covalent bonds and a lone pair are present, whereas three metavalent bonds and no lone pairs are the essential characteristics of crystalline Sb, crystalline Te being an intermediate case.

\section{Introduction}

The concept of lone pairs is frequently used to explain key features of the structure of molecules or of the atomic arrangement of certain solids. A lone pair refers to a pair of valence electrons that are not shared in a covalent bond with another atom. Lone pairs are also invoked to describe bonding in molecular crystals, from low temperature solid oxygen to ring, chain or planar structures of several other elemental or molecular solids. Sulfur, selenium or transition metal dichalcogenides adopt such structures upon crystallization. ${ }^{[1]}$ As the lone pairs are not involved in actual covalent bonding 
between atoms, the cohesion of the pseudomolecular constituents is then ensured by noncovalent, van der Waals (vdW) forces, and a van der Waals gap is observed. Yet, lone pairs are also discussed for chalcogenides with cubic rocksalt structure, such as PbTe or PbSe. In those cases, the lone pairs (if present) are obviously more difficult to detect, since the atomic arrangement is undistorted and vdW gaps are absent.

In some classes of chalcogenides, the ability to switch rapidly from a crystal to an amorphous phase that has very different optical and electrical properties is employed for data storage. These materials are called Phase Change Materials, and some of the most popular compounds are based on compounds along the pseudo-binary line between GeTe and $\mathrm{Sb}_{2} \mathrm{Te}_{3}\left(\mathrm{GeTe}, \mathrm{Ge}_{1} \mathrm{Sb}_{2} \mathrm{Te}_{4}, \mathrm{Ge}_{2} \mathrm{Sb}_{2} \mathrm{Te}_{5}, \ldots\right.$, $\left.\mathrm{Sb}_{2} \mathrm{Te}_{3}\right)^{[2]}$ These alloys often form layered structures in their most stable crystalline state, sometimes explained using lone pairs and $\mathrm{vdW}$ gaps. ${ }^{[3-7]}$ The amorphous structure, on the contrary, appears to be very different. ${ }^{[8-13]}$ Nevertheless, in some cases, lone pairs are also invoked to explain the structure of the amorphous phase, as in GeTe. ${ }^{[14]}$ Sometimes, the crystal's vdW gap is observed to reorganize under an electric field. Such an effect might provide an explanation for electronic switching in interfacial phase change memories. ${ }^{[15}$, 16]

More recently, an alternate description of a number of chalcogenides has been proposed. They have been qualified as metavalently bonded. The classification of metavalent bonding as a novel, fundamental bonding mechanism, is based on a unique combination of properties [17] and an unconventional bond rupture upon exposition to high electrical fields in conjunction with short, intense, laser pulses. ${ }^{[18]}$ Using the two quantumchemical coordinates, the electrons transferred between adjacent atoms and the electrons shared between them, a map can be spanned, ${ }^{[19,20]}$ which separates the different bonding mechanisms (metallic, ionic and covalent). Interestingly, metavalent solids are located in a well-defined region between ionic, covalent and metallic bonding, as confirmed recently by other quantum level descriptors ${ }^{[21]}$.

Hence, we have a number of chalcogenides including $\mathrm{Te},{ }^{[22]} \mathrm{Sb}_{2} \mathrm{Te}_{3},{ }^{[23]} \mathrm{Bi}_{2} \mathrm{Se}_{3}, \mathrm{Bi}_{2} \mathrm{Te}_{3},{ }^{[24,}$ 25]

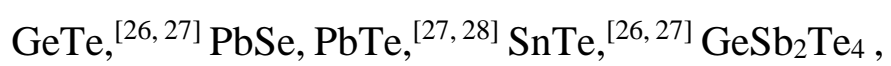
$\mathrm{Ge}_{2} \mathrm{Sb}_{2} \mathrm{Te}_{5}$ (GST225), ${ }^{[29]} \mathrm{AgSbTe}_{2}$ and related compounds, ${ }^{[30]}$ where both lone pairs and metavalent bonding have been suggested to be responsible for technologically important material properties. Since lone pairs and metavalent bonding correspond to two quite different types of electron localization mechanisms, it is thus necessary to derive unambiguous indicators for their presence and relative importance.

Fortunately, in modern quantum chemistry tools have been developed in recent years that provide a quantitative description of bonding in solids. In this work, we have used those tools to obtain a coherent view of bonding in crystalline chalcogenides and related compounds by addressing 5 prototypical examples, $\mathrm{PbO}, \mathrm{Se}, \mathrm{Te}, \mathrm{Ge}_{2} \mathrm{Sb}_{2} \mathrm{Te}_{5}$ and $\mathrm{PbTe}$. We will show that several of these systems show clear evidence of the presence of lone pairs, while others do not.

Using first principle wavefunction approaches, lone pairs can be identified by performing an analysis of the charge density and its topology. ${ }^{[31]}$ Most of the time, lone pairs can be associated to a well identifiable pocket of localized electrons. This pocket is characterized by negative values of the Laplacian of the density around a local minimum of this function. The so-called Electron Localization Function (ELF ${ }^{[32]}$ ) provides a measure of the degree of electron localization at each point of the system, relative to that of the uniform electron gas sharing the same electron density value at that point. Lone pairs appear as areas of high ELF value, due to the fact that the kinetic energy density is high in those areas in comparison with a homogeneous gas at the same electron density. ${ }^{[33]}$ This can be seen in Figure 1, where ELF isosurfaces with a high value represent well the shape of the highly localized valence charge density pockets (see Figure 2). The number of electrons involved into those localization regions (so-called $f$-localization domains ${ }^{[34]}$ ) depends critically upon the threshold $f$ used. By definition, the ELF value varies between 0 and 1 . A value of 1 is obtained for a complete localization and a value of 0.5 is obtained if the kinetic energy is that of the uniform electron gas. 


\section{WILEY-VCH}

(a)

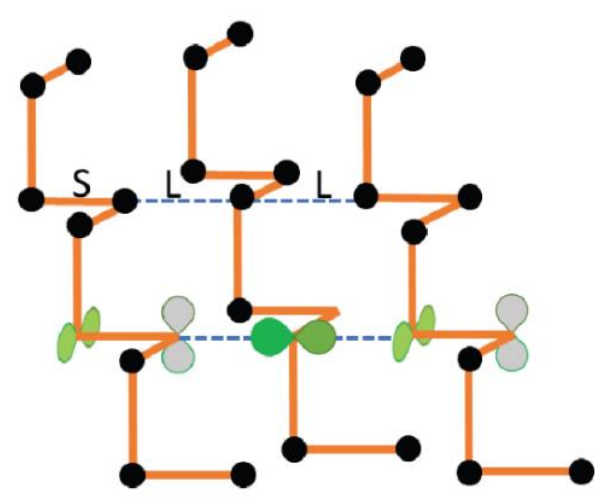

(b)

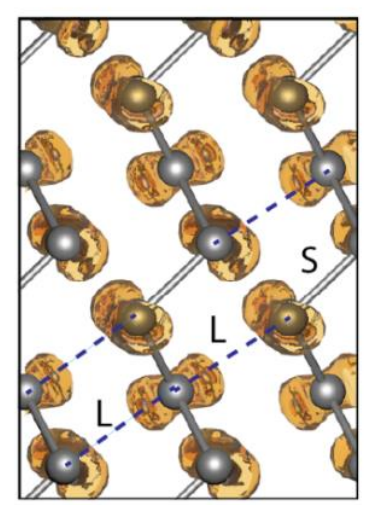

(c)

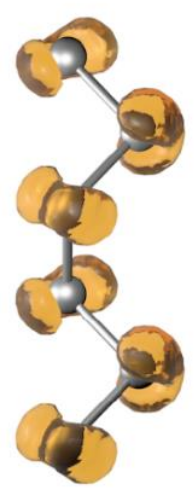

Figure 1 : Structure and Electron Localization Function of crystalline Selenium. (a) Schematic representation of the crystal structure. It is derived from a simple cubic structure and produced by a Peierls distortion that triples the unit cell, creating shortlong-long (SLL) bond alternation. Some non-bonding $p$ orbitals are represented in green. (b, c) $f$-localization ELF domains (not to be confused with charge density, i.e. Bader basins) plotted at the $f=0.85$ isovalue level. The ELF is computed using the valence charge density obtained through the VASP code with PAW potentials ${ }^{[35]}$ (b) along the (010) axis (c) isolated chain. The highly localized electron pockets (corresponding to the non-bonding $\mathrm{p}$ orbitals) are aligned with bonds on neighboring chains. The integrated charge density inside each pocket equals about 2 electrons. It is worth noticing that these high ELF value regions are obtained for the valence charge only and as such they refer to fictitious, yet well-defined and possibly useful, electron and kinetic energy density models. The inclusion of the $d$ electrons has a significant effect, lowering the ELF values as explained in Ref. ${ }^{[36]}$.

Classically, crystalline Se is described as a molecular crystal made of covalently bonded chains. Since Se has six valence electrons ( $2 s$ and $4 p$ electrons), two electrons remain non-bonding and form a lone pair. Therefore, Se forms chains and fulfills the $8-\mathrm{N}$ rule, the cohesion being due to dispersion forces. Alternatively, the twofold coordination has been explained by the Peierls distortion mechanism, ${ }^{[37,38]}$ that stabilizes the structure by opening a gap at the Fermi level. The structure can then be described as a short-long-long alternation of bonds in three (almost) orthogonal directions in space (see Figure 1). ${ }^{[37]}$ In three dimensions, this results in chains with welldefined relative orientation.

Thus, on each atom, two $p$ orbitals are involved in the formation of bonds, whereas the third one is left intact and creates a free pair of electrons. These electrons are not shared between atoms and highly localized, thus we can denote them as a lone pair. On the other hand, the alignment between the intra-chain bond and bonds on neighboring chains is in agreement with the Peierls distortion mechanism and it has been linked to the resonant bonding picture ${ }^{[39}$, ${ }^{40]}$ More recently, the metavalent bonding concept was introduced which can explain several effects ${ }^{[19]}$ found in GeTe and related systems, but not in resonantly bonded systems such as graphene and graphite.
Metavalent bonding has been introduced to explain the unconventional property portfolio of several chalcogenides including GeTe, $\mathrm{Sb}_{2} \mathrm{Te}_{3}, \mathrm{GeSb}_{2} \mathrm{Te}_{4}$, $\mathrm{Ge}_{2} \mathrm{Sb}_{2} \mathrm{Te}_{5}$, PbTe and related compounds. Since the concept of chemical bonding has created so much controversy, ${ }^{[41,42]}$ it is desirable to focus on observable quantities, which are related to chemical bonding. Indeed, a number of material properties including the effective coordination number $(\mathrm{ECON})$, the chemical bond polarizability, i.e. Born effective charge $Z^{*}$, the optical dielectric constant $\varepsilon_{\infty}$, as a measure of the electronic polarizability, and the Grüneisen parameter for transverse optical modes $\gamma_{\text {TO }}$ as a measure of the anharmonicity of the solid can be employed to separate ionic, metallic and covalent bonding. Interestingly, the chalcogenides listed above possess a property portfolio which differs from solids, which employ ionic, covalent or metallic bonding [17]. Furthermore, these chalcogenides also show an unconventional bond rupture upon laser-assisted field dissociation, as utilized in atom probe tomography. ${ }^{[18]}$ This is strong evidence that another, different bonding mechanism prevails in these solids. Interestingly, recent quantum chemical calculations provide a data-driven 
framework for computational screening and discovery of "metavalent" solids ${ }^{[21]}$. These findings all provide strong evidence for metavalent bonding as a novel, fundamental bonding mechanism in solids. Furthermore, quantum-chemical calculations reveal that metavalent bonding is accompanied by a particular range for two quantities, the number of electrons shared between adjacent atoms, which should be close to 1 , i.e. corresponding to a $2 \mathrm{c}-$ 1e bond, while the charge transfer between the atoms should be small to moderate. The view that metavalent bonding is a type of chemical bonding, distinctively different from ionic, covalent and metallic bonding is supported by the observation of distinct property changes upon crossing the border between covalent and metavalent solids. $^{[43]}$ Hence, covalent and metavalent solids can be distinguished unambiguously. In the past, chalcogenides like $\mathrm{GeTe}, \mathrm{Sb}_{2} \mathrm{Te}_{3}, \mathrm{GeSb}_{2} \mathrm{Te}_{4}$, and $\mathrm{Ge}_{2} \mathrm{Sb}_{2} \mathrm{Te}_{5}$ have been identified as being resonantly bonded. This wording which some of us have also employed and advocated ${ }^{[40,44,45]}$ is quite misleading though, since many scientists relate resonant bonding to the bonding mechanism in benzene, graphite or graphene. Graphite or benzene have properties such as the optical dielectric constant $\varepsilon_{\infty}$ or the Grüneisen parameter for transverse optical modes $\gamma_{\mathrm{TO}}$, which are very different from the properties of the chalcogenides listed above. ${ }^{[17]}$ In addition, graphene nanosheets show a
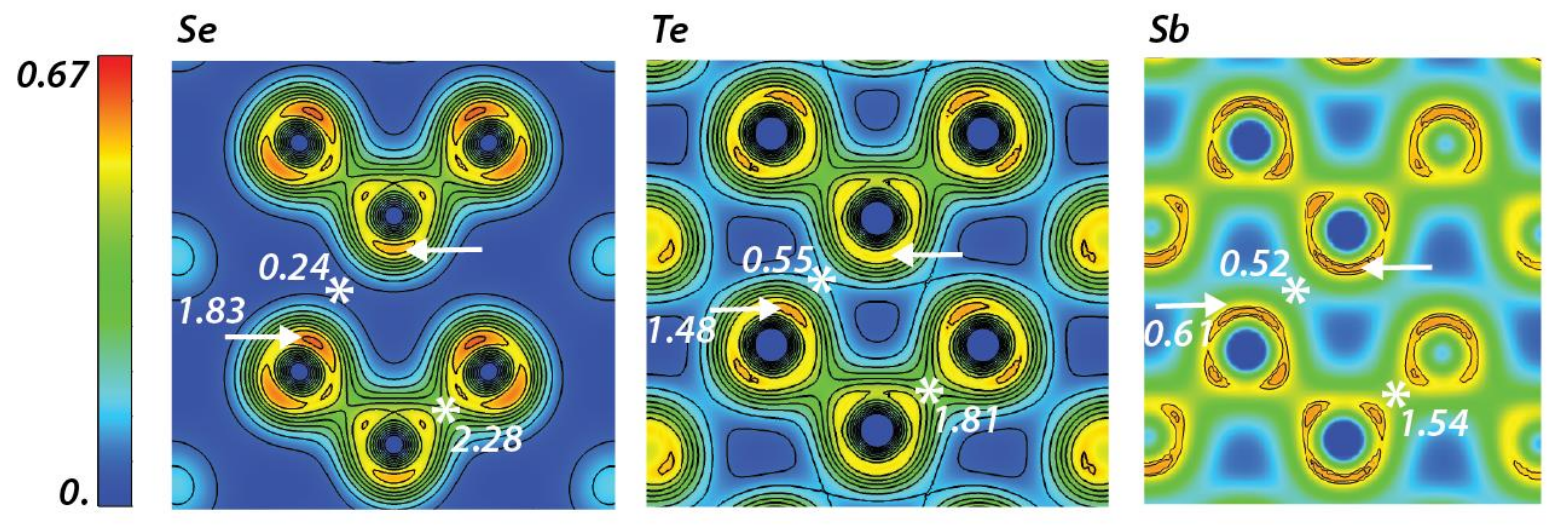

Figure 2: Valence charge density plots for Se, Te and Sb. Color scale from 0 to 0.67 e/ $/ \AA^{3}$ in Se and Te, but $0-0.47$ e/ $\AA^{3}$ for $\mathrm{Sb}$. The arrows denote high charge density areas where the valence ELF value is also large. The white asterisk lies in the middle of the $\mathrm{X}-\mathrm{X}(\mathrm{X}=\mathrm{Se}, \mathrm{Te}, \mathrm{Sb})$ intrachain and of the $\mathrm{X}-\mathrm{X}$ interchain distance. The numbers indicated in white are the number of electrons shared (ES) between neighboring basins. For the lone pairs, the numbers correspond to the fractional number of electrons obtained by integrating a $f$-localization basin defined by an isosurface ELF value equal to 0.85 (arbitrary choice). 


\section{WILEY-VCH}

\section{Pair Density Representation}

As stated before, one of the theoretical tools used by chemists to describe the chemical bond is the Quantum Theory of Atoms in Molecules (QTAIM). ${ }^{[31]}$ Attributing the charge located at some point in space to a given atom is not a trivial task. One elegant way to achieve this goal is to analyze the topology of the charge density. Bader's approach ${ }^{[31]}$ is to define the border of an atomic basin by the continuous surface with vanishing density gradient in the direction of the surface normal. As an example, some Bader basins determined with the $\mathrm{Yu}$ and Trinkle algorithm [48] are shown for the high temperature phase of $\mathrm{PbO}$ in Figure 3.

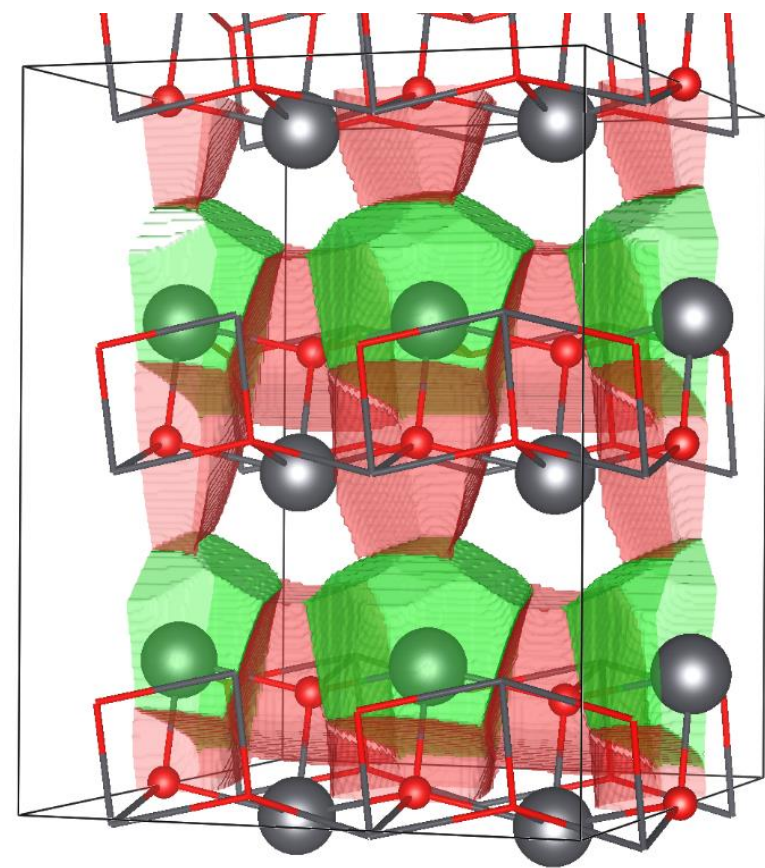

Figure 3 : Representation of some Bader basins in the layered massicot $\mathrm{PbO}$ structure (see text). The number of electrons shared is computed by choosing two different basins for the integration of the pair density. The number of transferred electrons is evaluated through integration of the electron density in the Bader basins.

The Bader charge is then obtained from the integration of the full charge density over an atomic basin $\Omega$. By comparing the Bader charges with those of the neutral atoms, one can obtain the number of electrons transferred (ET) between atoms.

Yet, the theory goes beyond the one-electron picture intrinsic to the charge density topology and introduces chemical bond descriptors in terms of explicit electron correlation effects. Particularly relevant are those defined in terms of the electron pair density. This pair density can be written as the product of two one-electron densities minus a correction, that is due to the exchange-correlation interaction.

$\rho\left(\boldsymbol{r}_{\mathbf{1}}, \boldsymbol{r}_{\mathbf{2}}\right)=\rho\left(\boldsymbol{r}_{\mathbf{1}}\right) \rho\left(\boldsymbol{r}_{\mathbf{2}}\right)-\rho\left(\boldsymbol{r}_{\mathbf{1}}\right) \rho\left(\boldsymbol{r}_{\mathbf{2}}\right) f\left(\boldsymbol{r}_{\mathbf{1}}, \boldsymbol{r}_{\mathbf{2}}\right)$

It thus expresses how the electronic density at a given point is modified by the correlation with an electron at another location. The second term in (5) is the exchange-correlation part, $\rho_{x c}\left(\boldsymbol{r}_{1}, \boldsymbol{r}_{2}\right)$, expressed with a correlation factor $f\left(\boldsymbol{r}_{1}, \boldsymbol{r}_{2}\right)$.

By integrating $\rho_{x c}\left(\boldsymbol{r}_{\mathbf{1}}, \boldsymbol{r}_{\mathbf{2}}\right)$ with coordinates $\mathbf{r}_{1}$ and $\mathbf{r}_{2}$ belonging to the same basin $\Omega$, one obtains the socalled localization index LI, which is a measure of the number of pair of electrons that are not exchanged with other basins. On the other hand, the integration over two electron coordinates, each spanning a different basin, yields the delocalization index DI, that measures the number of pairs of electrons shared (exchanged) between these two atomic basins $\Omega_{1}$ and $\Omega_{2}$. In this work, instead of employing the delocalization index, we will use ES $(=2 \mathrm{DI})$, the associated number of Electrons Shared in a 'covalent' way, between two atoms. ES hence provides a measure of the covalency of a bond. In the case of a lone pair of electrons, the two electrons are expected to be fully confined in the same atomic basin and contribute only to the localization index, LI. ${ }^{[49]}$ Alternatively, one can say that these would not contribute to bonding by delocalization with another basin, hence the lone pair will not contribute to ES. This analysis can thus help to identify both lone pairs and covalent bonds, as we will show in the following (note that pair density is clearly not available at the KS-DFT level, but a convenient, though thoroughly empirical, expression equal to the Hartree-Fock (HF) like ansatz for this quantity, is customarily adopted to 
evaluate the LIs and DIs. It is found that the KS-DFT LIs and DIs calculated in this way closely resemble the HF ones. The HF pair density takes full account of electron exchange correlation, while it totally neglects, as it is well known, electron coulomb correlation.

\section{Elemental crystals: Selenium, Tellurium and Antimony.}

In Figure 2, we compare the valence charge density (paw cores omitted) for crystalline $\mathrm{Se}, \mathrm{Te}$ and $\mathrm{Sb}$. Upon going from $\mathrm{Se}$ to $\mathrm{Te}$ and $\mathrm{Sb}$, the high density pockets become less prominent (with smaller curvature of the density) whereas electrons begin to populate the interchain space (white asterisk in Figure 2 ) finally forming a metavalent bond (see Ref. ${ }^{[19,50]}$ ). This trend is corroborated by the doubled electron density $\rho_{b}$ at the interchain bond critical point for Te and $\mathrm{Sb}$ ( $\rho_{\mathrm{b}}$ is $0.011 \mathrm{au}$ in Se and $0.020 \mathrm{au}$ in Te and $\mathrm{Sb})$. Correspondingly, the number of electrons shared between adjacent chains/planes, ES, increases from 0.24 in Se to 0.55 in Te and 0.52 in Sb.

Let us now use this approach to reinvestigate a few crystalline systems that have been previously linked to lone pairs.

\section{PbO : a well-known lone pair crystal.}

Crystalline $\mathrm{PbO}$ is considered as a prototype lone pair crystal ${ }^{[51]}$ in its $\alpha$-form (litharge structure). At high temperature, the massicot structure $(\beta-\mathrm{PbO})$ is stabilized. Both structures consist of stacked bilayers (see Figure 4), however the geometry is different enough to suggest rather different bonding characteristics. ${ }^{[52]}$

$\mathrm{PbO}$ has been studied intensively, in particular to understand the unusual atomic arrangement in the litharge structure in which layers of $\mathrm{Pb}$ atoms are facing each other. The $\mathrm{Pb}$ atoms form four bonds with $\mathrm{O}$ atoms located in the same plane. Such a configuration does not maximize the repulsion of negatively charged $\mathrm{O}$ atoms. The role of $\mathrm{Pb} 6 s$ electrons is essential for the formation of the structure, as they can hybridize with $\mathrm{O} 6 p$ orbitals to produce what is called a stereochemically active lone pair orbital pointing towards the interlayer space. ${ }^{[53-55]}$ These electrons contribute to the top of the valence band in both structures (see Figure 5).
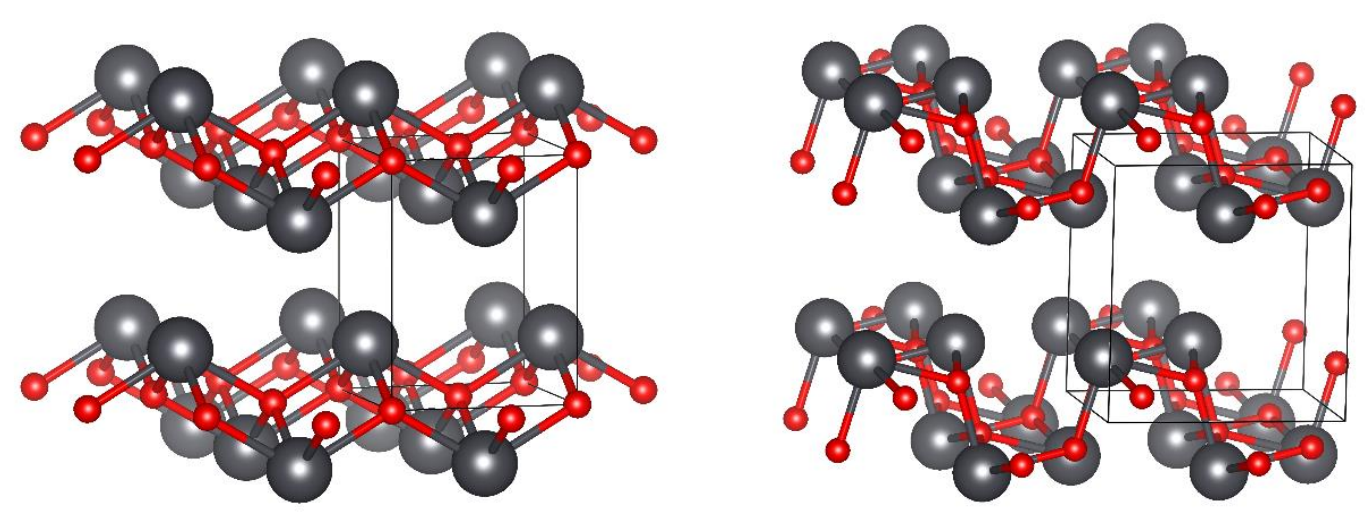

Figure $4: \mathrm{PbO}$ crystal structures. Left: low temperature $\alpha-\mathrm{PbO}$ (litharge) structure; Right: high temperature $\beta$ - $\mathrm{PbO}$ (massicot) structure. 


\section{WILEY-VCH}
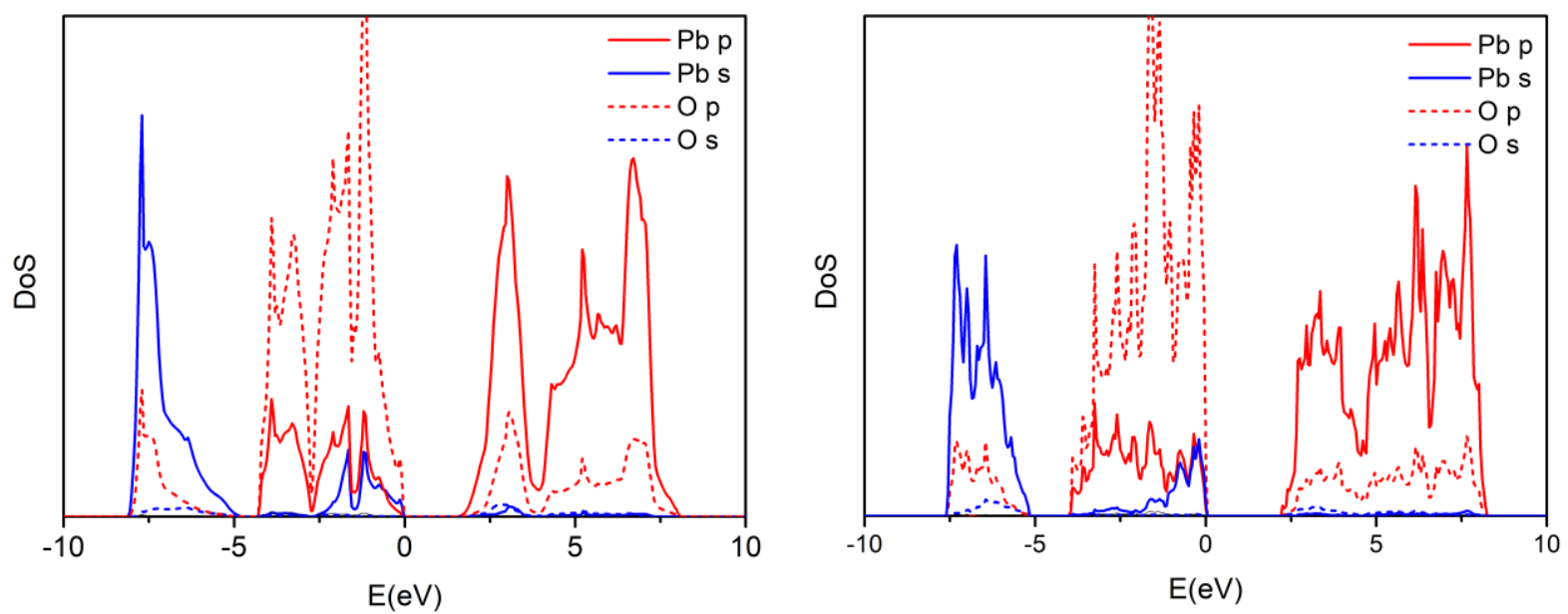

Figure 5 : Projected densities of states for litharge (left) and massicot (right). One can notice the contribution of $\mathrm{Pb} s$ states at the top of the valence band for both phases.

\section{Litharge}
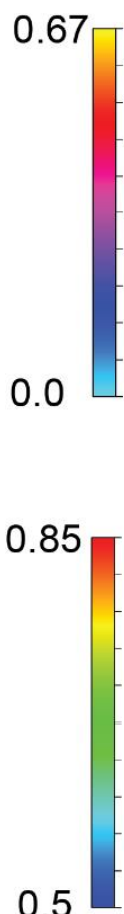
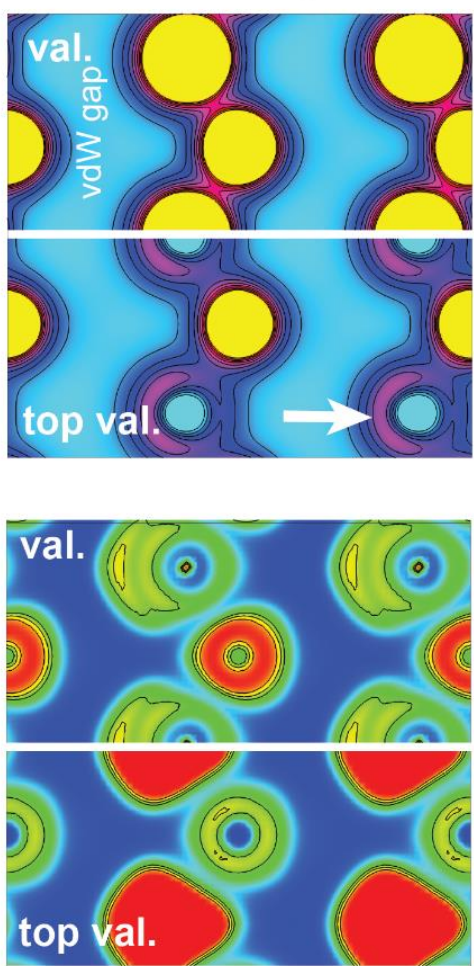

Massicot
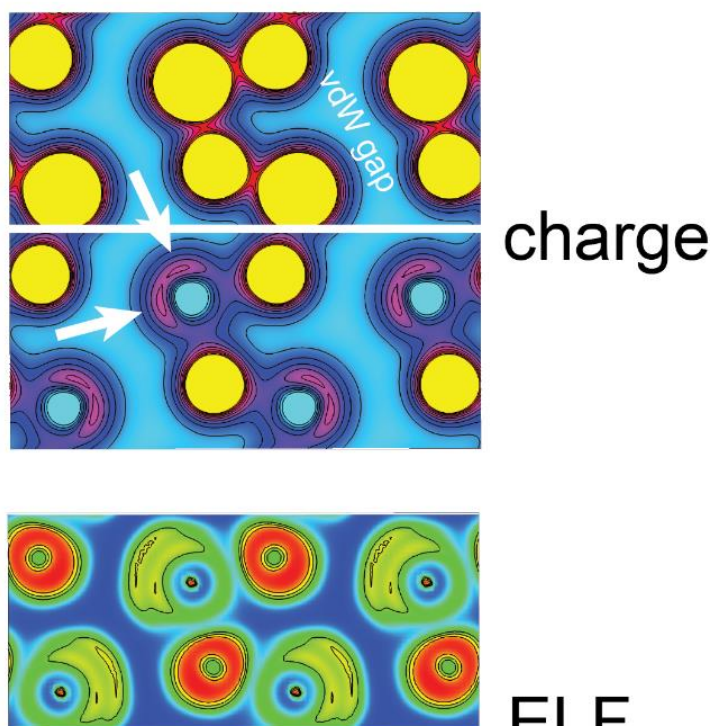

ELF

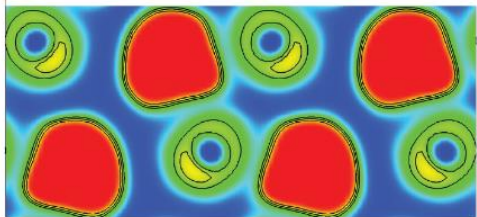

Figure 6 : Charge density (top) and ELF (bottom) contour plots for $\alpha-\mathrm{PbO}$ (left) and $\beta-\mathrm{PbO}$ (right) crystals. The planes are defined by $3 \mathrm{~Pb}$ atoms in both cases ( $\mathrm{O}$ atoms are slightly out-of-plane). Each panel is divided into two parts. The upper part is computed including all valence electrons, whereas the lower part is computed using only the upper valence states with energies ranging from $\mathrm{E}_{\mathrm{F}}-10 \mathrm{eV}$ to $\mathrm{E}_{\mathrm{F}}$. The color scale is $0-0.67 \mathrm{e} / \AA^{3}$ for the charge and $0.5-0.85$ for the ELF. In the present case, the charge density (top panels) does not show any charge accumulation between the layers. However, if only the states at the top of the valence bands are included in the charge density, then a slight increase of charge, highly localized, is seen in the interlayer spacing (white arrows). Similar conclusions can be drawn from the ELF panels. 
The inspection of the valence charge density (Figure 6) points out to the difficulty of identifying bonding electrons. Indeed, the high density of $\mathrm{Pb} d$ electrons gives the impression that the charge is essentially spherical around the $\mathrm{Pb}$ atoms. However, the $\mathrm{Pb} d$ electron state lie at much lower energy (out of scale in Figure 5). They do not contribute to the valence DOS down to $10 \mathrm{eV}$ below $\mathrm{E}_{\mathrm{F}}$ so that they can be safely considered as (pseudo) core states. When these are removed from the representation of the charge density, some electron pockets (arrows in Figure 6) are evidenced within the van der Waals gap. To measure the quantity of localized electrons, we use the ELF criterion to define our integration volume. Upon selecting the valence states (up from $\left.E_{F}-10 \mathrm{eV}\right)$, localized electrons, with large ELF values, appear very clearly (see Figure 7). If we select the same threshold that yields 2 electrons inside a lone pair in crystalline $\mathrm{Se}(\mathrm{ELF}=0.85)$, we obtain an integrated charge of about 1.3 electrons per $\mathrm{Pb}$ atom (Figure 7) for both phases. In the massicot structure, the localization pockets are a bit different. One can see in the valence charge density that small lobes appear on the opposite side of the $\mathrm{Pb}-\mathrm{O}$ bonds, giving the 1.3 electrons LP a more pronounced $p$ character. The band structure (Figure 8) further confirms that the $\mathrm{Pb} s$-states contribute to very weakly dispersive states around the valence band maximum (VBM), which accounts for the localized nature of LPs, but also the hybridization with the $\mathrm{O} p$ orbitals as described in Ref. ${ }^{[54]}$. It can be observed that the $\mathrm{Pb}-\mathrm{Pb}$ separation (4.09 and 4.19 $\AA)$ is slightly larger than the sum of the covalent radii $(4.04 \AA$ ) in both cases.
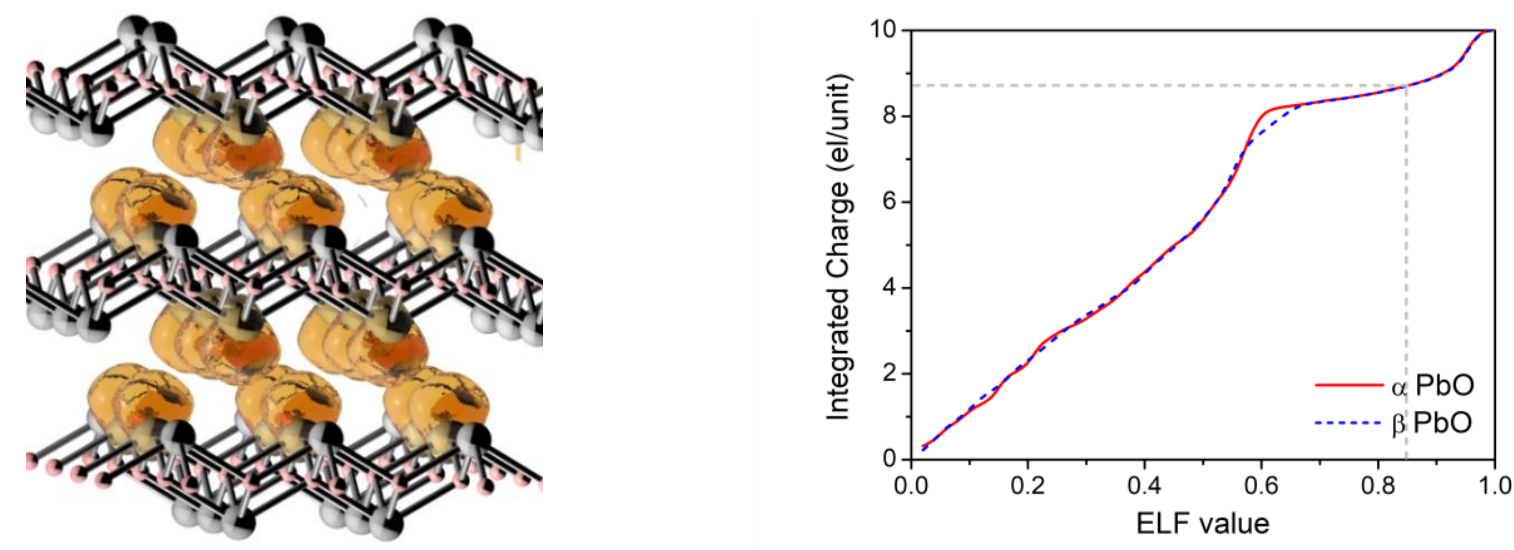

Figure 7 : Left, 3D view of the ELF 0.85 isosurface in the litharge structure; Right, integrated charge per Pb-O unit as a function of the ELF value in litharge and massicot. About 1.3 electrons are found inside each localization pocket $(f$-value between 0.85 and 1.0 ).
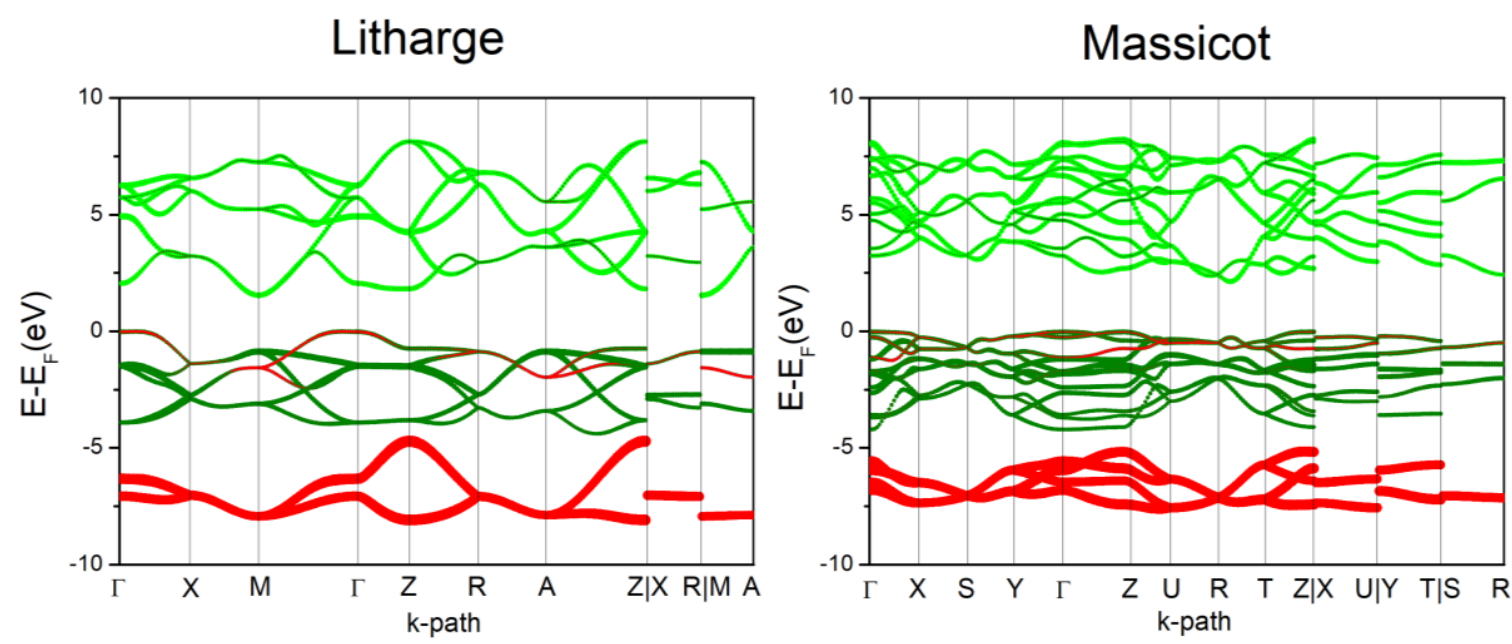

Figure 8: Fatband structure (lower states omitted) for $\alpha$-PbO (left) and $\beta$ - $\mathrm{PbO}$ (right) crystal. $\mathrm{Pb}$-states are colored in red, $\mathrm{O} p$-states in dark green and $\mathrm{Pb} p$ in light green. The size of the red lines is proportional to the contribution to the state. The bands overall appearance is similar (bandwidth). In particular, the top valence band are rather flat along the $\Gamma-\mathrm{Z}$ and $\Gamma-\mathrm{X}$ lines that is perpendicular to the layers in litharge and massicot, respectively. 
The Bader analysis (all Bader based calculations are performed on all electrons) shows that the massicot structure proves to be similarly ionic (1.17 electron transferred) to the litharge phase (1.16). But, let us now thus turn to the pair density analysis (see Table 1). In $\alpha$-PbO, 0.95 electrons are shared between $\mathrm{Pb}$ and $\mathrm{O}$ atoms, but almost no electrons are shared across the van der Waals gap. In $\beta-\mathrm{PbO}$, the $\mathrm{Pb}-\mathrm{O}$ bonding electrons are redistributed between the shorter and longer bonds arising from the distortion, but the number of shared electrons across the gap remains extremely small ( 0.03 and 0.05 electrons, respectively). This fact thus confirms that the localized electrons do not contribute to bonding at all, at least in a covalent way, since they do marginally contribute to the interlayer cohesion via van der Waals forces.

\begin{tabular}{|c|c|c|c|c|c|c|c|}
\hline \multirow{2}{*}{\multicolumn{2}{|c|}{$\begin{array}{l}\mathrm{PbO} \\
\text { Total Electron } \\
\text { Transfer }\end{array}$}} & \multicolumn{3}{|l|}{ Litharge } & \multicolumn{3}{|l|}{ Massicot } \\
\hline & & \multicolumn{3}{|l|}{$1.16 \mathrm{e}$} & \multicolumn{3}{|l|}{$101.17 \mathrm{e}$} \\
\hline \multirow{2}{*}{\multicolumn{2}{|c|}{ Electrons shared }} & $\begin{array}{lr}4 \mathrm{x} & \mathrm{Pb}-\mathrm{O} \\
4 \mathrm{x} & \mathrm{Pb}- \\
\mathrm{Pb} & \end{array}$ & $\begin{array}{l}2.35 \AA \\
3.73 \AA\end{array}$ & $\begin{array}{l}0.95 e \\
0.03 e\end{array}$ & $\begin{array}{l}1 \times \mathrm{Pb}-\mathrm{O} \\
1 \times \mathrm{Pb}-\mathrm{O} \\
2 \times \mathrm{Pb}-\mathrm{O}\end{array}$ & $\begin{array}{l}2.26 \AA \\
2.29 \AA \\
2.51 \AA\end{array}$ & $\begin{array}{l}1.16 \mathrm{e} \\
1.10 \mathrm{e} \\
0.72 \mathrm{e}\end{array}$ \\
\hline & & & & & \multicolumn{3}{|c|}{ Other $(\mathrm{r}>4.19 \AA)<\mathbf{0 . 0 5 e}$} \\
\hline LI & $\begin{array}{l}\mathrm{Pb} \\
\mathrm{O}\end{array}$ & $\begin{array}{l}11.61 \\
5.9\end{array}$ & & & $\begin{array}{l}11.63 \\
5.96\end{array}$ & & \\
\hline
\end{tabular}

Table 1 : Results of the pair density analysis in litharge and massicot. LI is the localization index. The LP contributions are all embedded in the LI, and the amount of electrons shared across the vdW gap $(3.73 \AA$ and $4.19 \AA$ wide $)$ is negligible.

\section{PbTe: a cubic thermoelectric compound.}

$\mathrm{PbO}$ is a well-known lone pair solid, yet with about only one localized valence electron per $\mathrm{Pb}(1.3 \mathrm{e}$ to be more precise), as defined at the ELF 0.85localization level. Nevertheless, the situation becomes less obvious when $\mathrm{O}$ is substituted by a heavier chalcogen such as Se or Te. In those cases, the stable structure is of $\mathrm{NaCl}$ type. This structure has been shown to be correlated with, if not responsible for, a number of enhanced or anomalous properties. Let us cite the high anharmonicity that is responsible for the high thermoelectric performance, ${ }^{[56]}$ extremely large Born effective charges and large dielectric constant, which leads to
$\mathrm{PbTe}$ being classified as an 'incipient metal'. ${ }^{[17]} \mathrm{On}$ the other hand, Bozin et al. demonstrated that there exists an underlying hidden symmetry breaking associated to local structural dipoles ${ }^{[57]}$ which have been linked to lone pairs. ${ }^{[58]}$ More recently, a comparative study of lead chalcogenides revealed that bonding in $\mathrm{PbTe}$ (and $\mathrm{PbSe}$ and $\mathrm{PbS}$ ) was significantly different from that in $\mathrm{PbO} .{ }^{[28]}$ The smaller difference in electronegativity in $\mathrm{PbTe}$, confirmed by the smaller electron transfer between $\mathrm{Pb}$ and $\mathrm{Te}$, together with the presence of about one electron in $\sigma p$-bonds, is not only causing the anomalous properties, but also appears to change the way bonds are breaking in atom probe measurements. This had PbTe classified as a 'metavalent' compound according to a recent classification. ${ }^{[19]}$ However, it is also commonly 


\section{WILEY-VCH}

stated that the physico-chemical behavior of $\mathrm{PbTe}$ is linked to lone pairs, ${ }^{[30]}$ although $p$-orbital overlap was shown recently to be responsible for the valence band dispersion [59] therefore questioning the relevance of lone pairs. Figure 9 shows a valence charge density (only $s$ and $p$ electrons) that is very much localized onto the atoms' cores, in agreement with the charge transfer of 0.7 electrons. The $p$ character of the bonds is more clearly evidenced around Te atoms. The ELF shows that these Te $p$ states are rather localized, but some $s$-like states appear localized on $\mathrm{Pb}$ atoms as well. If one would use the same criterion to define lone pairs in c-Se (iso-ELF value larger than 0.85), one would find about 2.7 localized electron per PbTe pair (mostly selectrons localized around each $\mathrm{Pb}$ atoms).
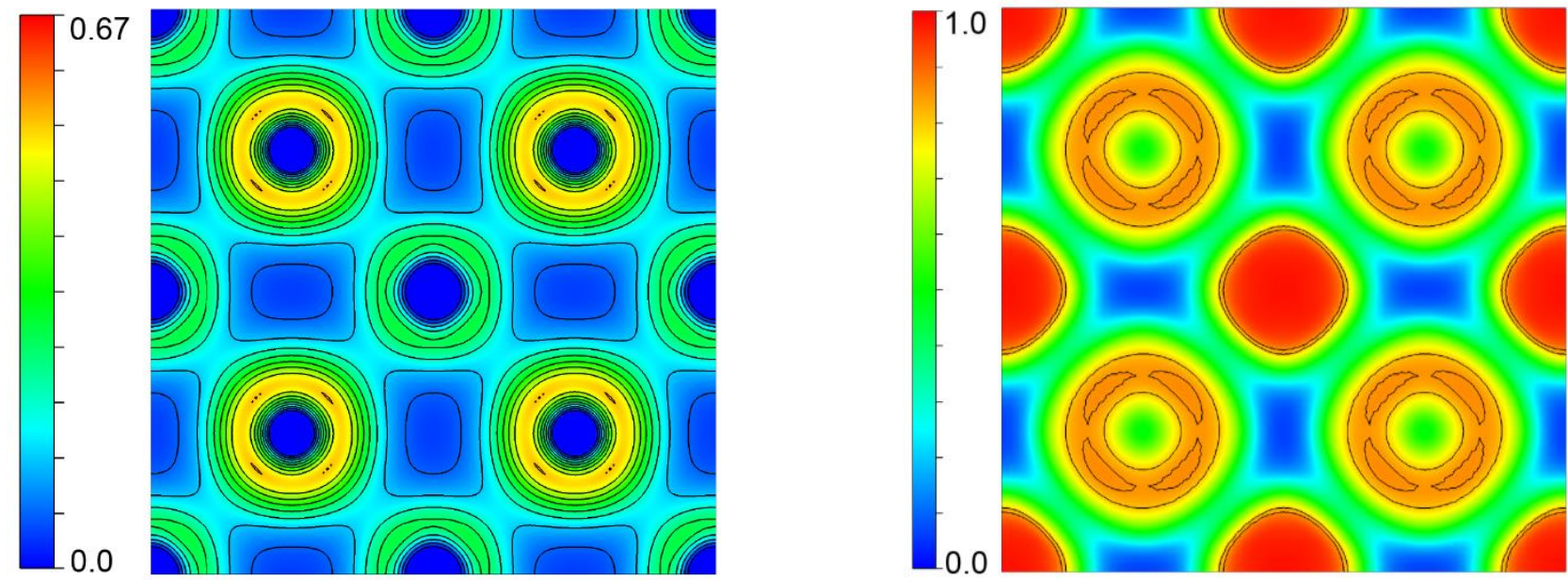

Figure 9 : Valence charge density (paw cores omitted, left) and valence ELF (right) for PbTe. The representation is made in the $<100>$ plane. The pseudocore $d$ electrons were included in the self-consistent calculation, but not in these two plots. Pb atoms are at the center of the figures.

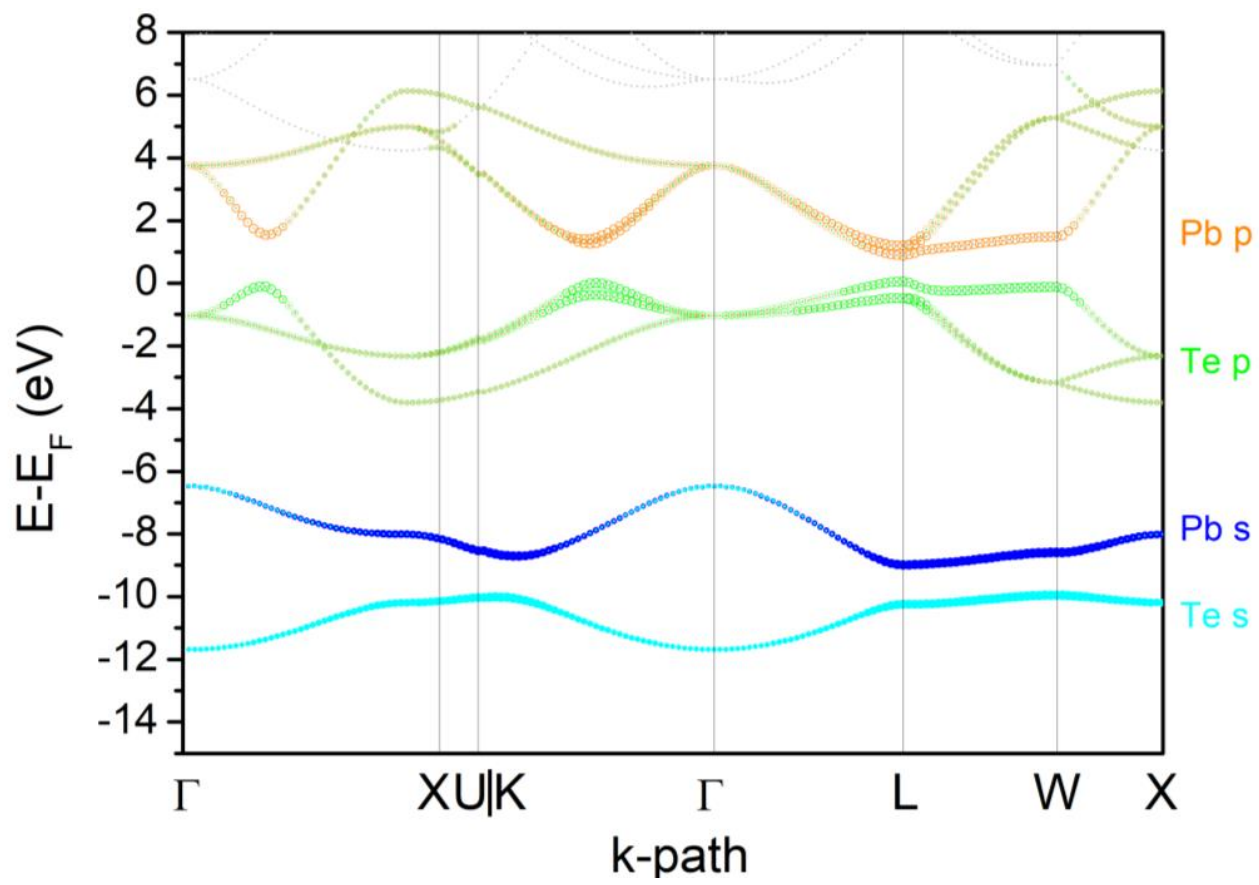

Figure 10 : Fatband (orbital-projected) structure for PbTe. The size of the symbols corresponds to the orbital contribution to the states. 
The band structure shown in Figure 10 shows that the $\mathrm{Pb} s$-states do not play any role in the uppermost valence states. They form a band centered about $8 \mathrm{eV}$ below $\mathrm{E}_{\mathrm{F}}$ in which they slightly hybridize with some Te $s$-states. This is totally different from the $\mathrm{PbO}$ structures, which indicate that $\mathrm{Pb} s$-orbitals in $\mathrm{PbTe}$ are not forming lone pairs, but some core-like states.

The case of the $p$-orbitals of $\mathrm{Te}$ is also very instructive. Figure 10 shows a global mirror symmetry between valence and conduction bands close to $\mathrm{E}_{\mathrm{F}}$. Only the relative contribution is inverted, Te $p$-electrons dominating the valence states whereas $\mathrm{Pb} p$-states dominate in the conduction band.

This is in line with the works by Walsh et al., ${ }^{[60]}$ in which the weak $s$ contribution at the top of valence in SnTe is linked to the higher energy of the Te-p orbital. This explains the absence of stereoactive lone pairs in SnTe whereas these appear in $\mathrm{SnS}$ and $\mathrm{SnSe}$. Besides, the relevance of lone pairs for thermoelectricity and optical properties in $\mathrm{Pb}-\mathrm{X}$ systems has been questioned recently ${ }^{[28,59,61]}$ as $p$ $p$ bonding appears to drive the essential features for these applications. We can also notice that the dispersive nature of the top valence and bottom conduction bands is due to the dominant role of $\mathrm{p}$ sigma bonding. Holm and coworkers showed that upon forcing a distortion, PbTe exhibits small localized lone pairs ${ }^{[58,62]}$ as in the stable GeTe phase. ${ }^{[27,63]}$ This is an important observation to understand the good thermoelectric properties of PbTe. However soft TO modes can be seen as an early sign of the onset of the Peierls distortion acting on a cubic network of pure p-sigma bonds. ${ }^{[64]}$

The analyses of the charge density and of the pair density data are consistent. Bader $(0.70 \mathrm{e})$ and Mulliken charges $(0.38 \mathrm{e})$ are different, but more importantly, the Mulliken analysis shows that each atom possesses about $2 s$ electrons, each $p$-orbital being filled by $0.56(\mathrm{~Pb})$ and $1.48(\mathrm{Te})$ electrons. A total of 6.12 electrons are contributing to six bonds, thus leaving 1.02 electrons per PbTe bond, not so different from the pair density value of 0.8 shared electrons.

Upon comparing $\mathrm{PbO}$ and $\mathrm{PbTe}$ (see Table 2), one can see that in the more ionic compound, lone pairs play a major role and no electrons are shared across a true van der Waals gap. On the contrary, when the charge transfer decreases, the electron sharing becomes dominant.

\section{6. $\mathrm{Ge}_{2} \mathrm{Sb}_{2} \mathrm{Te}_{5}$, a phase change material.}

Among the most studied layered chalcogenide crystals, one finds the Ge-Sb-Te alloy family. Indeed, these have been at the origin of the development of new types of non-volatile memories, based upon Phase Change Materials in which the two bits can be stored into a more conductive crystalline phase and a more resistive amorphous phase. ${ }^{[2,65,66]}$ However, it should be noted that it is not the stable crystal phase that is involved in that process, but a metastable cubic phase in which $\mathrm{Ge}$, $\mathrm{Sb}$ and vacancies are randomly sharing one site of the rocksalt structure. On the contrary, the stable crystal phase has been suggested as being crucial for a potential interfacial phase change memory. ${ }^{[67]}$ The stable crystal phase is known to be layered, with groups of 9 layers stacked in the z-direction of an hexagonal cell (Figure 11). Theoretically, the most stable arrangement, known as the Kooi structure (hereafter labeled GST225-Kooi), contains layered sequence Te-Sb-Te-Ge-Te-Ge-Te-Sb-Te. It has been argued that the 9 layers stacks are bonded to each other through a van der Waals gap (dashed line in Figure 11) in which lone pairs are responsible for the large interlayer distance. However, the nature of the van der Waals gap has been questioned in several papers. ${ }^{[24,68]}$ Similarly, the fact that strain can induce a change in properties ${ }^{[69,70]}$ seems incompatible with the existence of a true van der Waals gap. 

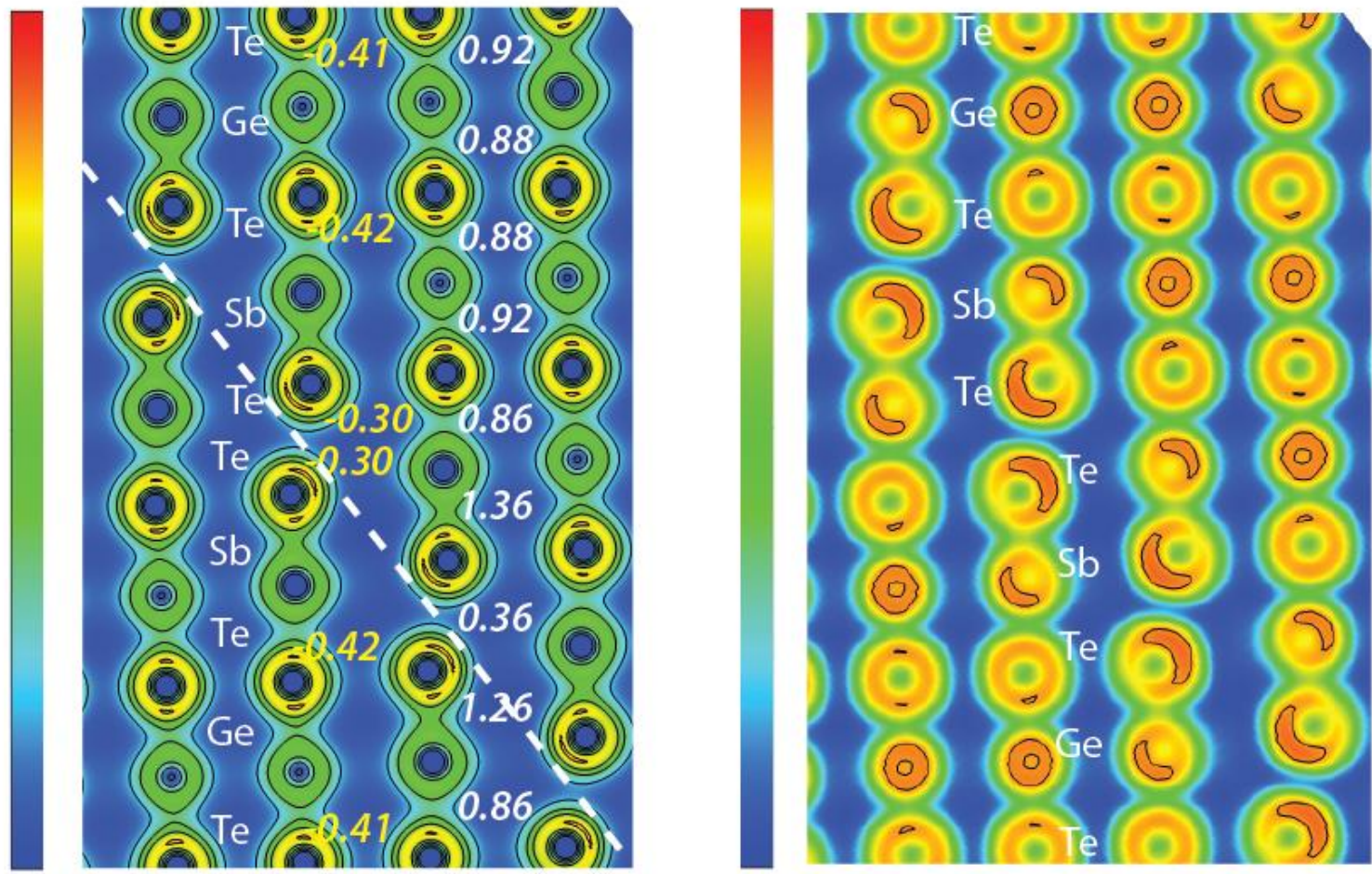

Figure 11: Valence charge density (paw cores omitted, left) and valence ELF for GST225-Kooi. The charge color scale goes from 0 to 0.67 . On the right panel, the ELF color scale goes from 0 to 1 with a contour line drawn at the 0.85 iso-level. Noticeable ELF contributions can be seen on $\mathrm{Ge}, \mathrm{Sb}$ atoms as well as on Te atoms located at the gap. Spherical localization regions are found around the $\mathrm{Ge}$ atoms, due to the s electrons. The white numbers on the left panel correspond to the number of electrons shared in the bonds, the Te atoms negative charges are indicated in yellow.

It should also be noted that the structure has been be explained by a Peierls distortion mechanism, ${ }^{[37]}$ in which a linear pattern of $\sigma$-bonded $p$-orbitals, occurring in 3 decoupled directions is stabilized by a distortion that multiplies the unit cell by a factor of nine. Figure 11 shows this distortion, with a zone depleted in electrons between Te layers at the edges of the nine layer group. At this point, it should be noted that the Te-Te distance across this gap is significantly smaller than expected, in line with previous reports which question the van der Waals bonding across the gap. ${ }^{[70]}$

This is consistent with our finding that there are indeed localized electrons in the structural gap (see Figure 11). Their appearance reminds of the $\alpha-\mathrm{PbO}$ case. Further inspection shows that opposite to that localized pocket within the gap, there is a pocket on the neighboring $\mathrm{Sb}$ atom, in addition to another spherical pocket (a pseudo core $s$ state, actually, as indicated by the lobster ${ }^{[71]}$ charge analysis) on the $\mathrm{Ge}$ atoms. Interestingly, the orbital decomposition of the charge gives similar numbers for all Te atoms, irrespective of their location (central or peripheral to the 9-layer stack). The detailed electron count indicates that there are about 4 electrons with
ELF $>0.85$ for the entire cell, with a large contribution from the Ge $s$-states. The number of electrons in the Te localization pocket is thus much smaller than one. Remarkably, all Te atoms have $1.9 s$-electrons and about 1.39 electron in each $p$ orbital, whereas $\mathrm{Sb}$ atoms have $1.95 s$-electron and 0.93 electron in each $p$-orbital (Ge: $1.9 s$ and 0.69 in each $p$-orbital). One can also notice that the charge transfer is thus quite moderate as measured from the charge density. Interestingly, the Bader charge of the Te atoms proves to be different according to the location of the atom (see Figure 11), the edge Te atoms being slightly less charged (-0.30e),

More importantly, and contrarily to both $\mathrm{PbO}$ crystal structures, in the case of GST, a small, but non-negligible fraction of electrons ( 0.36 electrons) is shared between pairs of Te atoms located on both side of the 'van der Waals' gap, which accounts for the reduced distance (see Table 2) when compared to twice the van der Waals radius of the Te atom. The all electron charge density analysis indicates the presence of a bond critical point (noted $(3,-1)$ ) at the center of the interlayer spacing, on the Te-Te segment and with a $\rho_{b}$ value $(0.015 \mathrm{au})$ which is 
exactly in between of those observed for Se and $\mathrm{Te}$ (see earlier).

These findings are comparable to what is observed in GeTe and many other compounds. ${ }^{[19,24,63,68,72]}$ In those cases, the interlayer separation is even smaller, which has been attributed to the metavalent nature of the bonds. ${ }^{[68]}$ In the case of GST, we nevertheless do not observe a normal 2c-1e bonding, as the alignment of charge concentrations is quite imperfect, thus leading to 0.3-0.4 electrons shared. It is therefore an intermediate case in which a pattern of self-avoiding lone pairs can be observed ${ }^{[72]}$ besides metavalent bonding. This conclusion is compatible with the unusual properties of GST225, such as the extremely high computed dielectric constant and the very large Born effective charges as well as well as the small electronic band gap. It is even suspected from these values that the system would easily become metallic under an electric field applied in the $\mathrm{z}$ direction. Recently, the existence of crystalline metavalent bonding has been shown to favor a faster kinetics of crystallization from the amorphous phase. ${ }^{[73]}$ The band structure shown in Figure 12 indicates that the $p$-orbitals of the edge Te atoms contribute to very dispersive valence and conduction states. This is also true along the very short $\Gamma$-A path, that is oriented along the c-axis of the hexagonal cell. The $s$-orbitals on these atoms do not hybridize with the $p$-states and are located at much lower energies.

\begin{tabular}{|l|l|l|l|l|l|}
\hline & ES & TET & Gap $(\AA)$ & $\sum$ r.vdW $(\AA)$ & Dev \\
\hline Se & $\begin{array}{l}2.28(2 \mathrm{x}) \text { intra } \\
0.24(4 \mathrm{x}) \text { inter }\end{array}$ & - & 3.57 & 3.80 & $-6 \%$ \\
\hline $\mathrm{Te}$ & $\begin{array}{l}1.81(2 \mathrm{x}) \text { intra } \\
0.56(4 \mathrm{x}) \text { inter }\end{array}$ & - & 3.51 & 4.12 & $-15 \%$ \\
\hline $\mathrm{Sb}$ & $\begin{array}{l}1.45(3 \mathrm{x}) \text { intra } \\
0.52(3 \mathrm{x}) \text { inter }\end{array}$ & - & 3.45 & 4.12 & $-19 \%$ \\
\hline $\mathrm{a}-\mathrm{PbO}$ & $\begin{array}{l}0.95(4 \mathrm{x}) \text { intra } \\
0.03(4 \mathrm{x}) \text { inter }\end{array}$ & 1.16 & $\begin{array}{l}4.09(\mathrm{~L}) \\
4.19(\mathrm{M})\end{array}$ & 4.04 & $\begin{array}{l}+1 \%(\mathrm{~L}) \\
+4 \%(\mathrm{M})\end{array}$ \\
\hline PbTe & $0.80(6 \mathrm{x})$ intra & 0.70 & 0.00 & 4.08 & - Inf. \\
\hline GST225 & $\begin{array}{l}1.36(3 \mathrm{x}) \text { intra } \\
0.36(3 \mathrm{x}) \text { inter }\end{array}$ & 0.3 & 3.65 & 4.12 & $-12 \%$ \\
\hline
\end{tabular}

Table 2 : Results of the pair density analysis and analysis of the interlayer spacing. ES is the number of electrons shared, with the multiplicity and the nature of the bonds (intra = intralayer, inter = across the vdW gap). TET is the total number of electrons transferred (charge). The gap is given by the shortest distance across the interlayer spacing. The deviation (dev) between the sum of van der Waals radii ( $\Sigma$ r.vdW ) and the gap is given in \%. For GST225, the selected atom is the Te atom at the van der Waals gap. For $\mathrm{PbO}$, L stands for litharge and $\mathrm{M}$ for massicot. 


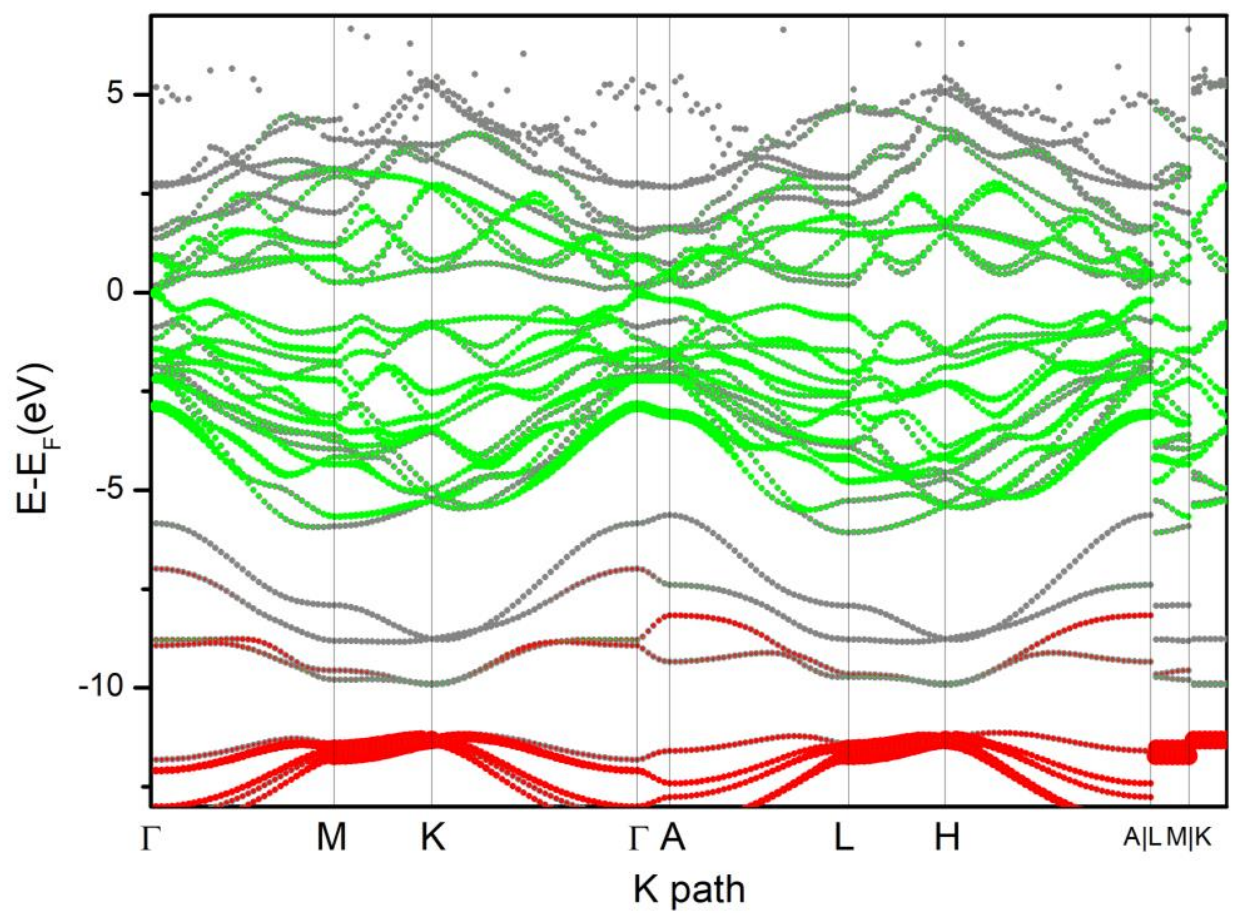

Figure 12 : Fatband plot for $\mathrm{Ge}_{2} \mathrm{Sb}_{2} \mathrm{Te}_{5}$ with the Kooi structure. The $s$ - and $p$-states corresponding to the Te atoms located around the gap are shown in red and green, respectively, their radius being proportional to the orbital contribution to the KS state.

\section{Conclusion.}

Lone pairs are often invoked to explain the peculiar behavior of certain solids, in particular when symmetry breaking occurs, such as in GST or when disorder is prevalent like in amorphous GeTe. In this work, we have shown that using Bader's approach and computing the electron pair density, it is possible to verify if lone pairs are indeed present. Lone pairs, as observed in $\alpha$ - and $\beta-\mathrm{PbO}$, can be evidenced in the band structure (very weakly dispersive state due to the localized nature of the LP), but also by the fact that no (or very few) electrons are shared in the space where LP's are observed as localized electron pockets in the valence ELF. In this case cohesion occurs via dispersion forces across a van der Waals gap. Its size is usually comparable to the sum of the atom's van der Waals radii. To actually measure whether electrons are shared across the possible vdW gap, it is required going beyond the one electron density provided by DFT, by analyzing the electron correlations. The QTAIM provides a robust tool, through the use of the pair density, to measure the degree of electrons shared between species. This shows that in PbTe metavalent bonding dominates over the impact of a very weak, electron-depleted $s$ lone pair. It also shows that layered GST crystal is an intermediate case where both misaligned lone pairs and metavalent bonding coexist, which accounts for the reduced van der Waals gap width. The Selenium crystal structure proves to be at the edge of a metavalently bonded system. Indeed, localized valence electron pockets containing 2 electrons are clearly identified, i.e. a lone pair prevails, yet some electrons are nevertheless shared between atoms located on neighboring Se chains. These are not enough to be able to classify Se as a metavalent crystal, ${ }^{[19]}$ in contrast to Te.

\section{Methods}

The ab Initio calculations were performed in the framework of Density Functional Theory (DFT). Three different plane wave basis codes were employed, including ABINIT, ${ }^{[74,75]}$ VASP $^{[76]}$ and PWSCF. ${ }^{[7]}$ The VASP and ABINIT codes were used in conjunction with PAW potentials ${ }^{[35,78]}$ and 
PBE exchange correlation, ${ }^{[79]}$ PWSCF was applied with norm-conserving ${ }^{[80]}$ and PAW potentials together with PBE. ${ }^{[81]}$ The PBESOL functional ${ }^{[82]}$ has been shown to provide improved structural parameters for layered $\mathrm{Ge}_{\mathrm{x}} \mathrm{Sb}_{\mathrm{y}} \mathrm{Te}_{\mathrm{z}}$ crystals. ${ }^{[83]}$

The structures have been relaxed to less than $1 \mathrm{E}-$ $5 \mathrm{eV} / \mathrm{A}$ and the plane wave cutoff was chosen large enough to ensure convergence of the energy to less than $1 \mathrm{meV} /$ atom. The initial DFT wavefunctions have then been post-processed either in the DGRID code ${ }^{[84]}$ or followed by a transformation into maximally localized Wannier functions ${ }^{[85,86]}$ and integration ${ }^{[48]}$ within the CRITIC2 code. ${ }^{[87]}$ In those calculations, the full density (charge + valence) is analyzed to define the Bader volumes.

\section{Acknowledgements}

J.-Y.R. acknowledges the collaborative convention between CEA-LETI and FRS-FNRS. M.W. acknowledges support by the DFG (SFB 917). J.Y.R. acknowledges computational resources provided by the CECI funded by the F.R.S.-FNRS under Grant No. 2.5020.11 and the Tier-1 supercomputer of the Fédération WallonieBruxelles, infrastructure funded by the Walloon Region under grant agreement $n^{\circ} 1117545$. J.Y. R acknowledges the FNRS CDR 'ABIGLO' grant J.0154.21. We acknowledge the computational resources granted from RWTH Aachen University under project RWTH0508 (initially JARA0183 and JARA0198).

Received: ((will be filled in by the editorial staff))

Revised: ((will be filled in by the editorial staff)) Published online: ((will be filled in by the editorial staff))

\section{References}

[1] M. Kulichenko, A. I. Boldyrev, J. Phys. Chem. C 2020, 124, 6267.

[2] M. Wuttig, N. Yamada, Nat. Mater. 2007, 6, 824.
[3] A. Lotnyk, U. Ross, T. Dankwort, I. Hilmi, L. Kienle, B. Rauschenbach, Acta Mater 2017, 141, 92.

[4] R. Wang, V. Bragaglia, J. E. Boschker, R. Calarco, Crystal Growth \& Design 2016, 16, 3596.

[5] P. Kowalczyk, F. Hippert, N. Bernier, C. Mocuta, C. Sabbione, W. Batista-Pessoa, P. Noé, Small 2018, 14, 1704514 [6] F. d'Acapito, P. Kowalczyk, J. Raty, C. Sabbione, F. Hippert, P. Noé, J. Physics D Appl. Phys. 2020, 53, 404002.

[7] J. Zhou, Z. Sun, Y. Pan, Z. Song, R. Ahuja, Europhys. Lett. 2011, 95, 27002.

[8] J.-Y. Raty, C. Otjacques, J.-P. Gaspard, C. Bichara, Solid State Sci. 2010, 12, 193.

[9] J.-Y. Raty, C. Otjacques, R. Peköz, V. Lordi, C. Bichara, in Molecular Dynamics Simulations of Disordered Materials: From Network Glasses to Phase-Change Memory Alloys (Eds: C. Massobrio, J. Du, M. Bernasconi and P. S. Salmon), Springer, Cham, Switzerland, 2015, pp. 485-509.

[10] S. Caravati, M. Bernasconi, T. D. Kühne, M. Krack, M. Parrinello, Appl. Phys. Lett. 2007, 91, 171906.

[11] R. Mazzarello, S. Caravati, S. Angioletti-Uberti, M. Bernasconi, M. Parrinello, Phys. Rev. Lett. 2010, 104, 085503. [12] M. Krbal, A. V. Kolobov, P. Fons, J. Tominaga, S. R. Elliott, J. Hegedus, T. Uruga, Phys Rev B 2011, 83, 054203.

[13] J. Akola, J. Larrucea, R. Jones, Phys Rev B 2011, 83, 094113.

[14] T. H. Lee, S. R. Elliott, Adv. Mater.2020, 32, 2000340.

[15] A. V. Kolobov, P. Fons, Y. Saito, J. Tominaga, ACS Omega 2017, 2, 6223.

[16] Y. Saito, P. Fons, K. V. Mitrofanov, K. Makino, J. Tominaga, J. Robertson, A. V. Kolobov, Pure and Applied Chemistry 2019, 91, 1777.

[17] M. Wuttig, V. L. Deringer, X. Gonze, C. Bichara, J.-Y. Raty, Adv. Mater. 2018, 30, 1803777.

[18] M. Zhu, O. Cojocaru-Mirédin, A. M. Mio, J. Keutgen, M. Küpers, Y. Yu, J.-Y. Cho, R. Dronskowski, M. Wuttig, Adv. Mater. 2018, 30, 1706735.

[19] J.-Y. Raty, M. Schumacher, P. Golub, V. L. Deringer, C. Gatti, M. Wuttig, Adv. Mater. 2019, 31, 1806280.

[20] Y. Cheng, S. Wahl, M. Wuttig, Phys. Status Solidi RRL 2021, 15, 2000482.

[21] D. Giri, L. Williams, A. Mukherjee, K. Rajan, J. Chem. Phys. 2021, 154, 124105.

[22] S. Yi, Z. Zhu, X. Cai, Y. Jia, J.-H. Cho, Inorg. Chem. 2018 , 57, 5083.

[23] J. C. Martinez, L. Lu, J. Ning, W. Dong, T. Cao, R. E. Simpson, Phys. Status Solidi (b) 2020, 257, 1900289.

[24] Y. Cheng, O. Cojocaru-Mirédin, J. Keutgen, Y. Yu, M. Küpers, M. Schumacher, P. Golub, J.-Y. Raty, R. Dronskowski, M. Wuttig, Adv. Mater. 2019, 31, 1904316.

[25] E. B. Isaacs, G. M. Lu, C. Wolverton, J. Phys. Chem. Lett. 2020, 11, 5577.

[26] H. Wang, P. Gopal, S. Picozzi, S. Curtarolo, M. Buongiorno Nardelli, J. Sławińska, npj Comput. Mater. 2020, $6,7$.

[27] U. Waghmare, N. Spaldin, H. Kandpal, R. Seshadri, Phys. Rev. B 2003, 67, 125111.

[28] S. Maier, S. Steinberg, M. Schumacher, P. Golub, J.-Y. Raty, R. Nelson, R. Mazzarello, O. Conjocaru-Mirédin, R. Dronskowski, M. Wuttig, Adv. Mater.2020, 32, 2005533.

[29] B. J. Kooi, J. T. M. D. Hosson, J. Appl. Phys. 2002, 92, 3584. 
[30] M. D. Nielsen, V. Ozolins, J. P. Heremans, Energy Environ. Sci. 2013, 6, 570.

[31] R. F. W. Bader, Atoms in Molecules - A Quantum Theory, Clarendon Press, Oxford, UK 1994.

[32] B. Silvi, A. Savin, Nature 1994, 371, 683.

[33] R. J. Gillespie, E. A. Robinson, J. Comput. Chem. 2007, $28,87$.

[34] A. Savin, B. Silvi, F. Colonna, Can. J. Chem 1996, 74, 1088.

[35] G. Kresse, D. Joubert, Phys. Rev. B 1999, 59, 1758.

[36] M. Kohout, A. Savin, J. Comput. Chem. 1997, 18, 1431.

[37] J. Gaspard, F. Marinelli, A. Pellegatti, Europhys. Lett. 1987, 3, 1095.

[38] J. P. Gaspard, A. Pellegatti, F. Marinelli, C. Bichara, Phil. Mag. B 1998, 77, 727.

[39] B. Huang, J. Robertson, Phys. Rev. B 2010, 81, 081204.

[40] K. Shportko, S. Kremers, M. Woda, D. Lencer, J. Robertson, M. Wuttig, Nat. Mater. 2008, 7, 653.

[41] K. Gavroglu, A. Simões, Neither Physics nor Chemistry: A History of Quantum Chemistry, MIT Press, London,UK 2012.

[42] P. Ball, Nature 2011, 469, 26.

[43] L. Guarneri, S. Jakobs, A. von, S. Maier, M. Xu, M. Zhu, S. Wahl, C. Teichrib, Y. Zhou, O. Cojocaru-Mirédin, M. Raghuwanshi, C.-F. Schön, M. Drögeler, C. Stampfer, R. P. S. M. Lobo, A. Piarristeguy, A. Pradel, J.-Y. Raty, M. Wuttig, Adv. Mater. 2021, 2102356.

[44] D. Lencer, M. Salinga, B. Grabowski, T. Hickel, J. Neugebauer, M. Wuttig, Nat. Mater. 2008, 7, 972.

[45] G. Lucovsky, R. M. White, Phys. Rev. B 1973, 8, 660.

[46] M. Raghuwanshi, O. Cojocaru-Mirédin, M. Wuttig, Nano Letters 2020, 20, 116.

[47] S. Shaik, D. Danovich, J. M. Galbraith, B. Braïda, W. Wu, P. C. Hiberty, Angew. Chem. Int. Ed. 2020, 59, 984.

[48] M. Yu, D. R. Trinkle, J. Chem. Phys. 2011, 134, 064111. [49] X. Fradera, M. A. Austen, R. F. W. Bader, J. Phys. Chem. A 1999, 103, 304.

[50] M. Salinga, B. Kersting, I. Ronneberger, V. P. Jonnalagadda, X. T. Vu, M. Le Gallo, I. Giannopoulos, O. Cojocaru-Mirédin, R. Mazzarello, A. Sebastian, Nat. Mater. 2018, 17, 681.

[51] G. Trinquier, R. Hoffmann, J. Phys. Chem. 1984, 88, 6696. [52] H. J. Terpstra, R. A. de Groot, C. Haas, Phys. Rev. B 1995, $52,11690$.

[53] G. W. Watson, S. C. Parker, J. Phys. Chem. B 1999, 103, 1258.

[54] A. Walsh, G. W. Watson, J. Solid State Chem. 2005, 178, 1422.

[55] J. Berashevich, O. Semeniuk, J. A. Rowlands, A. Reznik, Europhys. Lett. 2012, 99, 47005.

[56] S. Lee, K. Esfarjani, T. Luo, J. Zhou, Z. Tian, G. Chen, Nat. Commun. 2014, 5, 3525.

[57] E. S. Božin, C. D. Malliakas, P. Souvatzis, T. Proffen, N. A. Spaldin, M. G. Kanatzidis, S. J. Billinge, Science 2010, 330, 1660 .

[58] K. A. U. Holm, N. Roth, C. M. Zeuthen, K. Tolborg, A. A. Feidenhans'1, B. B. Iversen, Phys. Rev. B 2020, 102, 024112. [59] M. K. Brod, M. Y. Toriyama, G. J. Snyder, Chem. Mater. 2020, 32, 9771.

[60] A. Walsh, D. J. Payne, R. G. Egdell, G.W. Watson, Chem. Soc. Rev. 2011, 40, 4455-4463.
[61] M. Cagnoni, D. Führen, M. Wuttig, Adv. Mater. 2018 30, 1801787.

[62] B. Sangiorgio, E. S. Bozin, D. C. Malliakas, M. Fechner, A. Simonov, M. G. Kanatzidis, S. J. L. Billinge, N. A. Spaldin, T. Weber, Phys. Rev. Materials 2018 2, 085402.

[63] J.-Y. Raty, M. Wuttig, J. Physics D Appl. Phys. 2020, 53, 234002.

[64] J. P. Gaspard, Phys. Status Solidi RRL 2021 , 15, 2000536.

[65] P. Noé, C. Vallée, F. Hippert, F. Fillot, J.-Y. Raty, Semicond. Sci. and Tech. 2017, 33, 013002.

[66] I. Friedrich, V. Weidenhof, W. Njoroge, P. Franz, M. Wuttig, J. Appl. Phys. 2000, 87, 4130.

[67] R. E. Simpson, P. Fons, A. V. Kolobov, T. Fukaya, M. Krbal, T. Yagi, J. Tominaga, Nat. Nanotechnol. 2011, 6, 501. [68] B. J. Kooi, M. Wuttig, Adv. Mater.2020, 32, 1908302.

[69] E. Zallo, S. Cecchi, J. E. Boschker, A. M. Mio, F. Arciprete, S. Privitera, R. Calarco, Sci. Rep. 2017, 7, 1466.

[70] R. Wang, F. R. L. Lange, S. Cecchi, M. Hanke, M. Wuttig, R. Calarco, Adv. Funct. Mater. 2018, 28, 1705901.

[71] S. Maintz, V. L. Deringer, A. L. Tchougréeff, R. Dronskowski, J. Comput. Chem. 2016, 37, 1030.

[72] K. Tolborg, C. Gatti, B. B. Iversen, IUCrJ 2020, 7, 480.

[73] C. Persch, M. J. Müller, A. Yadav, J. Pries, N. Honné, P. Kerres, S. Wei, H. Tanaka, P. Fantini, E. Varesi, F. Pellizzer, M. Wuttig, Nat. Comm. 2021, 12, 4978

[74] X. Gonze, G. M. Rignanese, M. Verstraete, J. M. Beuken, Y. Pouillon, R. Caracas, F. Jollet, M. Torrent, G. Zerah, M. Mikami, P. Ghosez, M. Veithen, J. Y. Raty, V. Olevano, F. Bruneval, L. Reining, R. Godby, G. Onida, D. R. Hamann, D. C. Allan, Z Kristallogr. 2005, 220, 558.

[75] X. Gonze, J. M. Beuken, R. Caracas, F. Detraux, M. Fuchs, G. M. Rignanese, L. Sindic, M. Verstraete, G. Zerah, F. Jollet, M. Torrent, A. Roy, M. Mikami, P. Ghosez, J. Y. Raty, D. C. Allan, Comput. Mater. Sci. 2002, 25, 478.

[76] G. Kresse, J. Furthmuller, Phys. Rev. B 1996, 54, 11169. [77] P. Giannozzi, S. Baroni, N. Bonini, M. Calandra, R. Car, C. Cavazzoni, D. Ceresoli, G. L. Chiarotti, M. Cococcioni, I. Dabo, A. Dal Corso, S. de Gironcoli, S. Fabris, G. Fratesi, R. Gebauer, U. Gerstmann, C. Gougoussis, A. Kokalj, M. Lazzeri, L. Martin-Samos, N. Marzari, F. Mauri, R. Mazzarello, S. Paolini, A. Pasquarello, L. Paulatto, C. Sbraccia, S. Scandolo, G. Sclauzero, A. P. Seitsonen, A. Smogunov, P. Umari, R. M. Wentzcovitch, J. Phys.: Condens. Matter 2009, 21, 395502.

[78] P. E. Blöchl, Phys. Rev. B 1994, 50, 17953.

[79] J. P. Perdew, K. Burke, M. Ernzerhof, Phys. Rev. Lett. 1996, 77, 3865.

[80] D. R. Hamann, Phys. Rev. B 2013, 88, 085117.

[81] C. Hartwigsen, S. Goedecker, J. Hutter, Phys. Rev. B 1998, 58, 3641.

[82] J. P. Perdew, A. Ruzsinszky, G. I. Csonka, O. A. Vydrov, G. E. Scuseria, L. A. Constantin, X. Zhou, K. Burke, Phys. Rev. Lett. 2008, 100, 136406.

[83] W. Ibarra-Hernández, J.-Y. Raty, Phys. Rev. B 2018, 97, 245205.

[84] M. Kohout, DGrid 5.1 2019

[85] N. Marzari, D. Vanderbilt, Phys. Rev. B 1997, 56, 12847. [86] A. A. Mostofi, J. R. Yates, Y.-S. Lee, I. Souza, D. Vanderbilt, N. Marzari, Comp. Phys. Commun. 2008, 178, 685. [87] A. Otero-de-la-Roza, E. R. Johnson, V. Luaña, Comp. Phys. Commun. 2014, 185, 1007. 
How to Identify Lone Pairs, van der Waals Gaps and Metavalent Bonding using Charge and Pair Density Methods: From Elemental Chalcogens to Lead Chalcogenides, Phase Change Materials and Hybrid Perovskites.

Jean-Yves Raty*1,2 , Carlo Gatti $^{3}$, Carl-Friedrich Schön ${ }^{4}$ and Matthias Wuttig 4,5,6

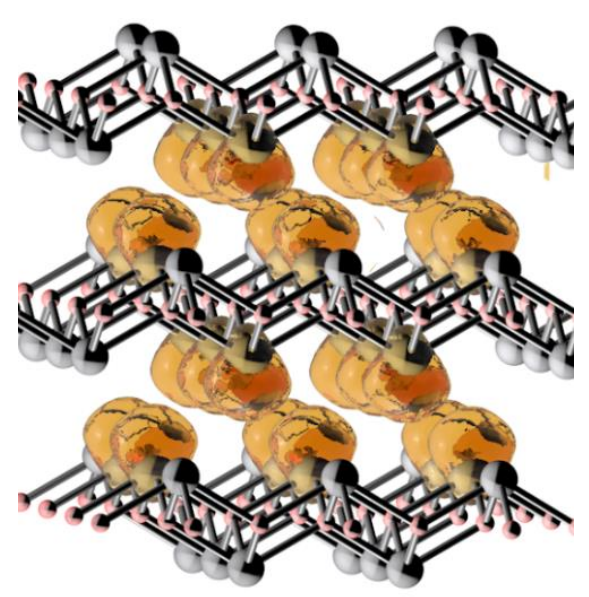

We present a method to assess the presence of lone pairs and van der Waals gaps in condensed systems by combining electron pair density method with regular charge density and ELF analysis. In particular, in metavalent systems, bonding electrons are responsible for absence of true van der Waals gaps. 